\title{
Modelagem dinâmica da expansão urbana usando autômatos celulares: 0 caso de Fortaleza-CE
}

\author{
Dynamic modeling of urban expansion using cellular automata: the case of \\ Fortaleza-CE
}

Laís Marques de Oliveira [a] [D] , Samíria Maria Oliveira da Silva [b] [D]

Francisco de Assis de Souza Filho [b] [iD, lana Alexandra Alves Rufino [c] [ib]

Higor Costa de Brito [d] (D), Tereza Margarida Xavier de Melo Lopes [a] (D)

[a] Universidade Federal do Ceará (UFC), Fortaleza, CE, Brasil

[b] Universidade Federal do Ceará (UFC), Departamento de Engenharia Hidráulica e Ambiental, Fortaleza, CE, Brasil

[c] Universidade Federal de Campina Grande, Unidade Acadêmica de Engenharia Civil, Campina Grande, PB, Brasil

[d] Universidade Federal de Campina Grande, Campina Grande, PB, Brasil

Como citar: Oliveira, L. M., Silva, S. M. O., Souza Filho, F. A., Rufino, I. A. A., Brito, H. C., \& Lopes, T. M. X. M. (2021). Modelagem dinâmica da expansão urbana usando autômatos celulares: o caso de Fortaleza-CE. urbe. Revista Brasileira de Gestão Urbana, v. 13. e20200092. https://doi.org/10.1590/2175-3369.013.e20200092

\section{Resumo}

Previsões do comportamento das mudanças que ocorrem no uso do solo são indispensáveis para um planejamento urbano adequado, indicando áreas mais propensas à ocupação humana. Dessa forma, o estudo tem por objetivo prever a expansão urbana da cidade de Fortaleza, localizada no nordeste do Brasil, utilizando um modelo dinâmico que combina autômatos celulares com as Cadeias de Markov (CA-Markov). Ele foi desenvolvido por meio de três etapas: aquisição e processamento dos dados; espacialização dos dados; e, modelagem dinâmica espacial. A matriz de transição markoviana forneceu o percentual de mudanças de uma classe para a outra ao algoritmo de simulação. Dados reais de entrada de uso do solo dos anos de 2009 e 2017 mostraram uma transição de $26,50 \%$ de áreas vegetadas para áreas urbanizadas. A validação do modelo apresentou excelentes resultados (índice de similaridade fuzzy superior a 0.8) para realizar simulações futuras (2020 até 2025). Segundo as previsões, em 2025, dos 297,10 km² de área urbanizada e vegetada, 82,43\% serão de área urbanizada e apenas 17,57\% serão de área vegetada. 0 modelo de simulação pode ser alimentado por mudanças que aconteçam dentro do período previsto, sendo incluídas na simulação como variáveis dinâmicas, obtendo uma simulação compatível com essas mudanças.

Palavras-chave: Urbanização. Modelagem dinâmica. Autômatos celulares.

\footnotetext{
LMO é mestre em Engenharia Civil e Ambiental, e-mail: lais.engciivil@gmail.com

SMOS é professora adjunta I, doutora em Engenharia Civil, e-mail: samiriamaria@gmail.com

FASF é professor associado, doutora em Engenharia Civil, e-mail: assissouzafilho@gmail.com

IAAR é professora associada, doutora em Recursos Naturais, e-mail: iana.alexandra@ufcg.edu.br

HCB é graduado em Engenharia Civil, e-mail: h_igor@hotmail.com

TMXML é graduanda em Engenharia Civil, e-mail: terezamelo@alu.ufc.br
} 


\section{Abstract}

Forecasts of the behaviour of changes that occur in land use are indispensable for adequate urban planning, indicating areas more prone to human occupation. Thus, the study aims to predict the urban expansion of the city of Fortaleza, located in northeastern Brazil, using a dynamic model that combines cellular automata with the Markov Chains (CA-Markov). It was developed through three steps: data acquisition and processing; spatialization of data; and, dynamic spatial modelling. The Markovian transition matrix provided the percentage of changes from one class to another to the simulation algorithm. Actual land use input data for the years 2009 and 2017 showed a transition of 26.50\% from vegetated to urbanized areas. The validation of the model showed excellent results (fuzzy similarity index greater than 0.8) to perform future simulations (2020 to 2025). According to forecasts, in 2025, of the $297.10 \mathrm{~km}^{2}$ of urbanized and vegetated area, $82.43 \%$ will be urbanized, and only $17.57 \%$ will be vegetated. The simulation model can be fed by changes that happen within the predicted period, included in the simulation as dynamic variables, obtaining a simulation compatible with these changes.

Keywords: Urbanization. Dynamic modeling. Cellular automata.

\section{Introdução}

A migração de populações para os centros urbanos é uma realidade. Na opinião de Martins et al. (2010), essa realidade se manterá no futuro e, se este pressuposto se confirmar, é bem possível que os equipamentos urbanos existentes não sejam capazes de absorver tamanhas demandas, sejam estas de água para consumo, capacidade de absorver as águas servidas e o lixo gerado ou ainda a capacidade de fluxo no sistema viário.

Esse crescimento sempre é acompanhado de mudanças no uso do solo que acarretam, de forma lenta ou rápida, a expansão da área urbanizada, que nos últimos anos levou a uma série de consequências ambientais negativas, como a poluição do ar, a formação de ilhas de calor urbano, a fragmentação da paisagem e a piora da qualidade de água (Li et al., 2017; Liu et al., 2019). Rubira (2016) corrobora com essa assertiva, ressaltando que a maioria das áreas verdes localizadas em municípios brasileiros, principalmente nos grandes centros populacionais, acaba sendo alterada negativamente pelo processo de expansão urbana acelerada e pelo crescimento populacional. Assim, compreender o espaço urbano e a evolução do seu padrão espacial torna-se imprescindível ao desenvolvimento social, econômico e ecológico dessas áreas.

Nesse sentido, as técnicas e os produtos compreendidos pelos sistemas de informações geográficas, sensoriamento remoto e modelagem dinâmica espacial têm sido um grande aliado ao desafio de planejar e ordenar o espaço urbano e seu entorno, proporcionando subsídios técnicos para a tomada de decisão dos planejadores e gestores (Stanganini \& Lollo, 2018). Para Rossetti et al. (2013), associar as tecnologias de sensoriamento remoto e geoprocessamento a modelos dinâmicos espaciais para avaliar de forma quantitativa a estruturação e a dinâmica do espaço urbano proporciona uma melhor visualização da realidade urbana e dos elementos responsáveis pelas suas transformações espaço-temporais.

Dentre as abordagens utilizadas na modelagem dinâmica têm-se os Autômatos Celulares (AC) que são capazes de modelar sistemas dinâmicos complexos tais como os sistemas urbanos. Para Votsis (2017), o uso de autômatos celulares é motivado pela capacidade de modelar a evolução da cidade concomitantemente aos impactos das intervenções espaciais e reproduzir a distribuição do crescimento de maneira espacialmente explícita, permitindo compreender as implicações das políticas espaciais alternativas e refiná-las.

Nesse sentido, o objetivo deste estudo é prever a expansão urbana da cidade de Fortaleza, localizada no Nordeste do Brasil, utilizando um modelo dinâmico que combina autômatos celulares com as cadeias de Markov (CA-Markov). Para isso, foram extraídas informações referentes ao uso do solo do 
Mapeamento Anual da Cobertura e Uso do Solo do Brasil (MapBiomas). Estas foram quantificadas com o auxílio da Linguagem de Programação R, através da interface RStudio, e modeladas por meio do software Dinâmica EGO.

\section{Modelagem Dinâmica Espacial}

A modelagem dinâmica espacial tem como base a modelagem de ecossistemas com extensões que acomodam a heterogeneidade espacial e os processos humanos de tomada de decisão. A principal característica dessa modelagem é a facilidade com que é utilizada na descrição da dimensão espacial, além do fato de descrever quantitativamente um fenômeno e prever sua evolução, integrando suas escalas temporal e espacial (Meirelles et al., 2007).

Uma das inúmeras utilidades da modelagem dinâmica é o entendimento das alterações do uso e cobertura da terra, possibilitando assim a realização de simulações de mudanças na paisagem como potencial ferramenta para antecipar prováveis tendências passíveis de ocorrerem em uma região (Almeida, 2003; Delaneze, 2011; Gonçalves et al., 2011; Maeda et al., 2011; Dias \& Walde, 2013).

A modelagem dinâmica pode ser realizada através do uso de "células". Esta abordagem tornou-se popular para fenômenos como propagação de doenças (Johansson et al., 2012), crime (Malleson \& Birkin, (2011) e do uso da terra (Matthews et al., 2007). Mais recentemente, Jat et al. (2017) apresentam uma possibilidade de integração de ferramentas do sensoriamento remoto, SIG e de um modelo baseado em autômatos celulares, o SLEUTH (do inglês: Slope, Land cover, Exclusion, Urbanization, Transportation, and Hillshade) de uma área urbana de maior complexidade devido a sua grande heterogeneidade.

Sakieh et al. (2015) utilizaram o modelo baseado em autômatos celulares SLEUTH para analisar a dinâmica da cidade de Karaj, capital da província de Alborz -Irã, e prever a tendência histórica do seu crescimento urbano. Segundo, Mas et al. (2014), mudanças no uso e cobertura do solo é um tópico que tem chamado bastante atenção de cientistas e tomadores de decisões. Nesse contexto, alguns estudos utilizando as ferramentas disponíveis na modelagem dinâmica espacial foram realizados para estudar e avaliar essas mudanças.

Maeda et al. (2011) utilizaram um modelo baseado em autômatos celulares (Dinâmica EGO) para analisar os processos de conversão florestal ocorridos no Parque Nacional do Xingu, obtendo bons resultados quanto à calibração e validação.

Com o intuito de estudar a dinâmica do uso da terra durante um período de 18 anos na cidade de Harbin, Gong et al. (2015) utilizaram SIG e modelos baseados em autômatos celulares e descobriram que houve perdas significativas nos usos da terra denominados "terras agrícolas", "pastagens", "terras úmidas" e "várzea" em favor de "terrenos construídos" e menores transformações de "várzea" para "área florestal" e "corpo de água".

Inicialmente, os conceitos de autômatos celulares foram apresentados por John Von Newmann. Em 1982, John Conway deu grande popularidade a esses conceitos, quando apresentou o Jogo da Vida, onde ele demonstrou que quando regras muito simples são aplicadas repetidamente sobre estados aleatórios, produzem resultados semelhantes à forma como certos sistemas evoluem no mundo real (Roy \& Snickars, 1996).

No Jogo da Vida, a representação do espaço é feita através de uma grade de células, podendo ser células vivas ou mortas. A partir de um estado inicial aleatório, a cada geração, novas células nascem, e algumas morrem. A vizinhança de cada célula determina seu estado; no caso do Jogo da Vida, essa vizinhança é definida por quatro células adjacentes.

Sobre cada célula de um autômato celular são aplicadas regras de transição (Figura 1), que determinam quando e por que o estado de uma célula se altera e podem ser qualitativas ou quantitativas (Almeida et al., 2008). De acordo com o que é especificado pelas regras de transição, o estado de uma célula no instante $t+1$ (futuro) é igual ao da sua vizinhança no instante $t$ (atual), se os vizinhos tiverem os 
estados iguais, caso contrário, o estado da célula permanece o mesmo. A partir disso, pode-se observar que as mudanças que ocorrem são estritamente locais, isto é, baseadas nas vizinhanças de cada célula.

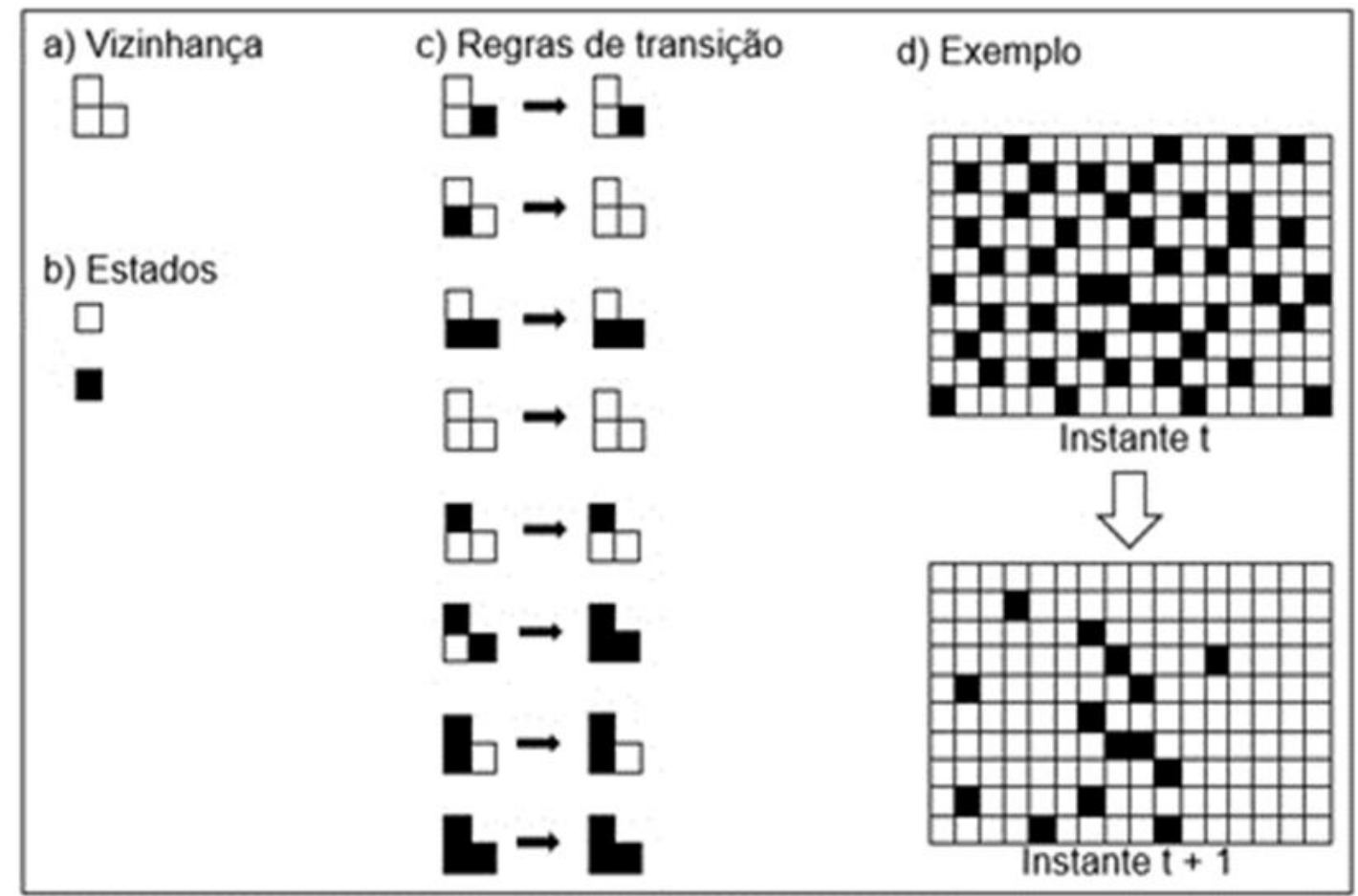

Figura 1 - Exemplo de regra de transição de autômato celular. Fonte: Câmara et al. (1996).

A Figura 1 apresenta os componentes básicos de um autômato celular clássico, sendo eles:

- Espaço euclidiano, dividindo em um array ${ }^{1}$ de células;

- Uma vizinhança de tamanho e formato definidos (Figura 1.a);

- Um conjunto de estados discretos (Figura 1.b);

- Um conjunto de regras de transição (Figura 1.c);

- Um conjunto de intervalos de tempo, com atualização simultânea das células (Figura 1.d).

\section{Área de Estudo}

O estudo é aplicado para a cidade de Fortaleza, capital do Estado do Ceará, que está localizada na Região Nordeste do Brasil (Figura 2). Segundo estimativas do IBGE (2018), esta cidade possui uma população de 2.643 .247 habitantes, com aproximadamente $312,407 \mathrm{~km}^{2}$ de área.

\footnotetext{
${ }^{1}$ Um array (ou arranjo) é uma estrutura de dados que armazena uma coleção de elementos de tal forma que cada um dos elementos possa ser identificado por, pelo menos, um índice ou uma chave.
} 


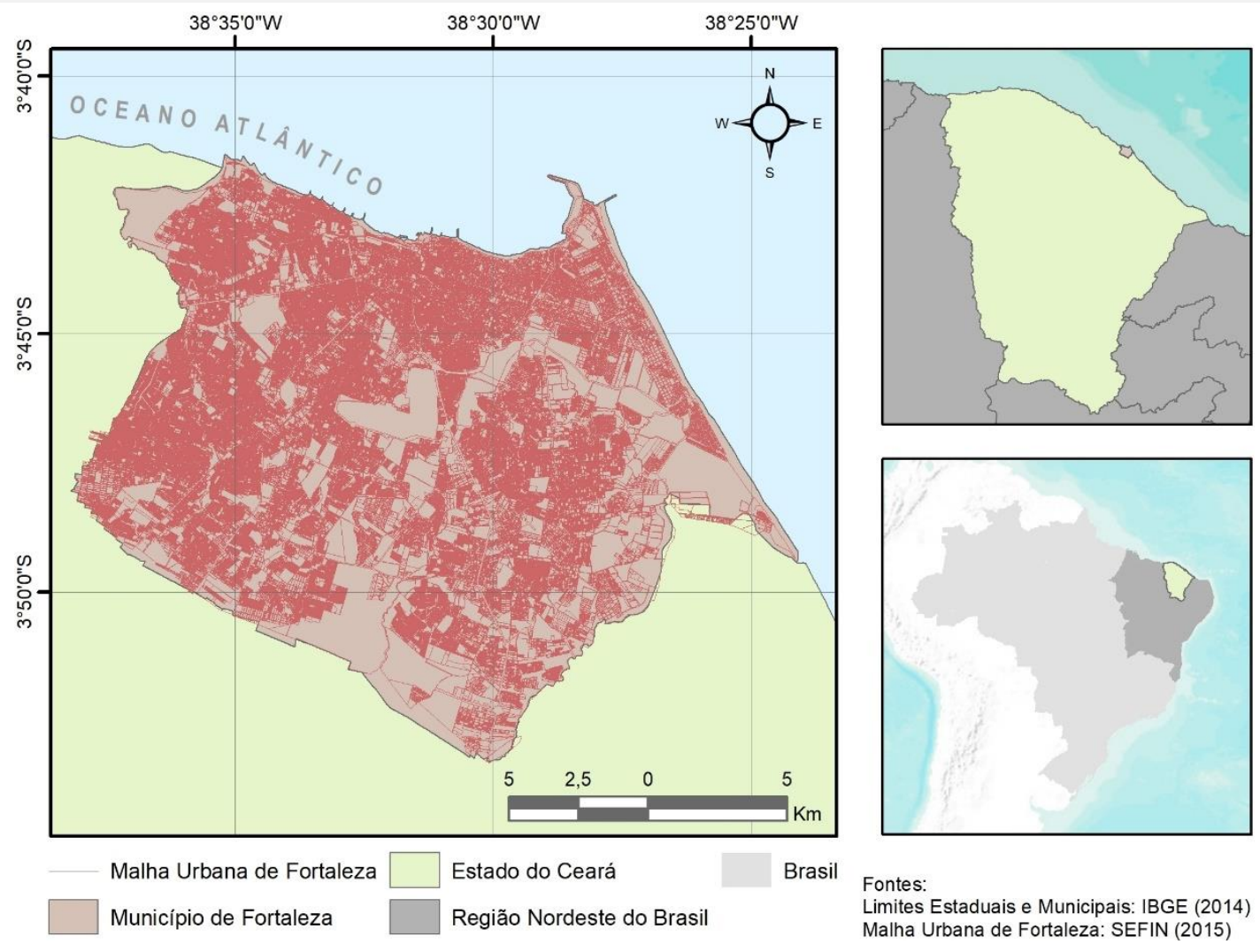

Figura 2 - Localização da capital do Ceará - Fortaleza. Fonte: Autores (2020).

O processo de ocupação de Fortaleza teve início na região central e se expandiu para oeste da cidade contornando o rio Pajeú. Esta aglomeração populacional no centro prevaleceu e se acentuou devido às imigrações advindas do interior do estado do Ceará por conta dos eventos de seca. Tal cenário se intensificou de maneira significativa a partir de 1866 quando o acesso à cidade foi ampliado por meio de ferrovias e rotas marítimas (Almeida \& Rosen, 1993).

A partir de então, a ocupação de Fortaleza deixou de ser monocentral e passou a ser policentral, crescendo de forma dispersiva. Atualmente, a distribuição espacial da cidade pode ser caracterizada como heterogênea e desordenada, na qual existem áreas de maior concentração populacional onde prevalecem comunidades humildes e zonas de maior poder aquisitivo que possuem menores aglomerações (IPLANFOR, 2015). Tal desordenamento, associado aos vazios urbanos, gera complicações para o planejamento e operação da infraestrutura existente.

\section{Metodologia}

Para o desenvolvimento do estudo adotaram-se três etapas: (i) aquisição e processamento dos dados; (ii) espacialização dos dados; e, (iii) modelagem dinâmica espacial (Figura 3). Cada uma dessas etapas foi discutida na sequência do texto. Na modelagem foi utilizado o software Dinâmica EGO (acrônimo para Environment for Geoprocessing Objects ou ambiente para geoprocessamento de objetos), desenvolvido pelo Centro de Sensoriamento Remoto da Universidade Federal de Minas Gerais (CSR/UFMG). Na espacialização dos dados foi utilizado o software QGIS. 


\section{ETAPAS METODOLÓGICAS}

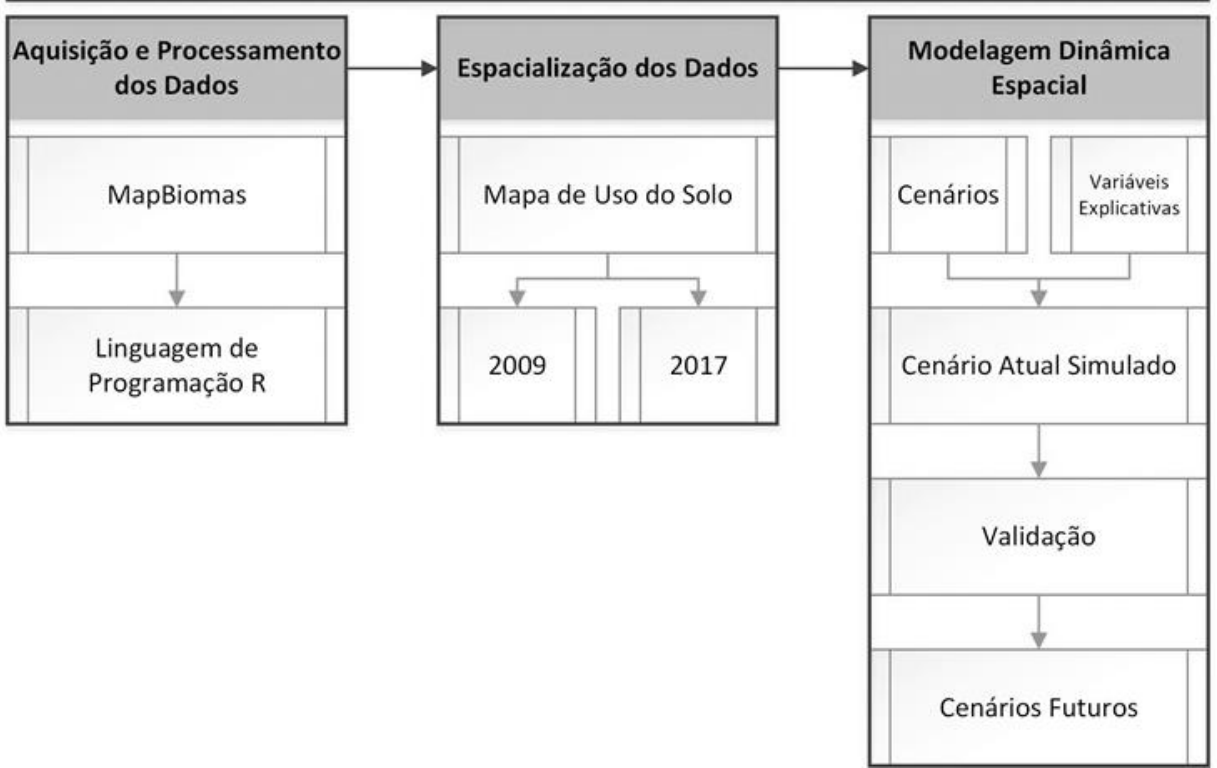

Figura 3 - Diagrama esquemático das etapas metodológicas. Fonte: Autores (2020).

\section{Aquisição, Processamento e Espacialização dos Dados de entrada do modelo de simulação}

Para a realização da modelagem dinâmica espacial utilizaram-se como dados de entrada mapas de uso do solo em tamanho matricial 30x30 metros, feitos a partir de mosaicos Landsat (5, 7 e 8) disponibilizados no MapBiomas, como também variáveis explicativas (estáticas e dinâmicas) das mudanças do uso do solo. A aquisição e processamento dos dados de uso do solo foram extraídos do MapBiomas, proposto por Souza \& Azevedo (2017), por meio da plataforma Google Earth Engine (Gorelick et al., 2017). Para a elaboração dos mapas em formato matricial, os dados do MapBiomas para os anos 2009 e 2017 foram quantificados através de uma tabela de frequência gerada na Linguagem de Programação R com auxílio da interface RStudio, onde o valor de cada pixel correspondia a uma classificação de uso do solo. Em seguida esses mapas foram espacializados, sendo reclassificados com o auxílio do QGIS em dois valores de pixel, valor de pixel 1 correspondendo a área vegetada e valor de pixel 2 correspondendo a área urbanizada.

As variáveis que descrevem as mudanças que ocorrem na área de estudo podem ser de dois tipos: variáveis dinâmicas e variáveis estáticas. As variáveis dinâmicas recebem esse nome porque são atualizadas ao longo da simulação, enquanto com as variáveis estáticas isso não ocorre. As variáveis dinâmicas são calculadas pelo próprio modelo de simulação e a variável estática foi escolhida para melhor explicar e informar ao modelo onde as mudanças ocorreram e quais mudanças ocorreram durante os oito anos em análise.

Portanto, foram considerados os seguintes cenários do uso do solo de Fortaleza para compor os dados de entrada do modelo de simulação: (i) Cenário I: referente ao ano de 2009; (ii) Cenário II: referente ao ano de 2017. Além dos mapas de entrada, variáveis explicativas (Quadro 1) foram inseridas no modelo.

Quadro 1 - Variáveis utilizadas na modelagem

\begin{tabular}{|ll|}
\hline Variável estática & Variáveis dinâmicas \\
\hline Mapa de diferença de uso do solo entre os anos 2009 e 2017 & Distância à áreas urbanizadas \\
\cline { 2 - 2 } & Distância à áreas vegetadas \\
\hline
\end{tabular}

Fonte: Autores (2020). 


\section{Modelagem Dinâmica Espacial - Calibração e Validação do Modelo de Simulação}

A calibração do modelo consistiu em quatro etapas: cálculo das matrizes de transição, cálculo dos coeficientes dos pesos de evidência, análise da correlação dos mapas, e ajuste e execução do modelo de simulação. A primeira etapa consiste na construção de uma matriz de probabilidade de transição Markoviana para cada uma das classes de uso do solo para os anos de 2009 e 2017, ao qual serve para fornecer o percentual de mudanças de uma classe para a outra ao algoritmo de simulação. Essa informação é obtida por meio da tabulação cruzada do mapa de classes inicial e final (Figura 4). A matriz de probabilidade de transição ou matriz Markoviana descreve um sistema que muda em intervalos discretos de tempo. As Cadeias de Markov são um processo estocástico ao qual a saída desse modelo é baseada na probabilidade de mudanças, P_ij, entre as classes de cobertura da terra (i e j) (Vick \& Bacani, 2019). Portanto, na composição de uma paisagem com dois usos do solo, a probabilidade de transição P_12 seria a probabilidade da classe de uso do solo 1 (área vegetada) no tempo t0 mude para o tipo de uso do solo 2 (área urbanizada) no tempo t1.

O método dos Pesos de Evidência é aplicado para produzir um mapa de probabilidades de transição, que representa as áreas mais favoráveis para mudança. Para categorizar as variáveis contínuas, são calculados intervalos nos mapas de distâncias, de forma semelhante a um fatiamento.

A única suposição necessária ao método dos Pesos de Evidência é que os mapas de entrada devem ser espacialmente independentes. Para testar esta independência é feita uma análise da correlação de mapas. Na opinião de Bonham-Carter (1994), um grupo de medidas pode ser aplicado para estimar esta suposição, como o teste de Cramer (V) e o Joint Information Uncertainty (Incerteza de Informação Conjunta - JIU). Esses índices operam com valores reais e percentuais, respectivamente, e avaliam o grau de dependência espacial entre pares de variáveis. Conforme Bonham-Carter (1994) quanto mais próximos de 1, maior a dependência espacial entre os pares de variáveis consideradas. Variáveis com correlação acima de 0.5 (50\%) devem ser desprezadas ou combinadas em uma terceira que substituirá o par correlacionado no modelo.

A parametrização do modelo também incluiu o ajuste para a execução do modelo de simulação. 0 Dinâmica EGO usa como regra local de Autômato Celular um mecanismo de transição composto por dois functores complementares de transição: Patcher e Expander. A função Patcher destinou-se a gerar novas manchas, por meio de um mecanismo de constituição de sementes, enquanto a função Expander respondeu pela expansão de manchas previamente existentes de uma determinada classe (Soares-Filho et al., 2002).

Além da definição da proporção entre Patcher e Expander, foi necessário definir o tamanho médio e a variância da área de mudança, além do índice de isometria. 0 tamanho médio, variância e isometria da área de mudança foram obtidos por meio da tabela de frequência obtida no software RStudio.

O desempenho do modelo foi validado a partir de similaridade fuzzy. 0 índice de similaridade fuzzy empregado neste trabalho foi criado pelo Centro de Sensoriamento Remoto da Universidade Federal de Minas Gerais (CSR/UFMG) e representa uma adaptação do índice de similaridade fuzzy criado por Hagen (2003). No caso particular deste trabalho, a comparação foi feita entre dois mapas-diferenças, resultantes, de um lado, da subtração entre o mapa final real e o mapa inicial, e de outro lado, entre o mapa final simulado e o mapa inicial (Figura 4). Adotou-se uma função de decaimento exponencial com tamanho de janela 11x11 e uma função de decaimento constante, calculada com os seguintes tamanhos de janelas: $1 \times 1,3 \times 3,5 \times 5,7 \times 7,9 \times 9$ e 11x11. 


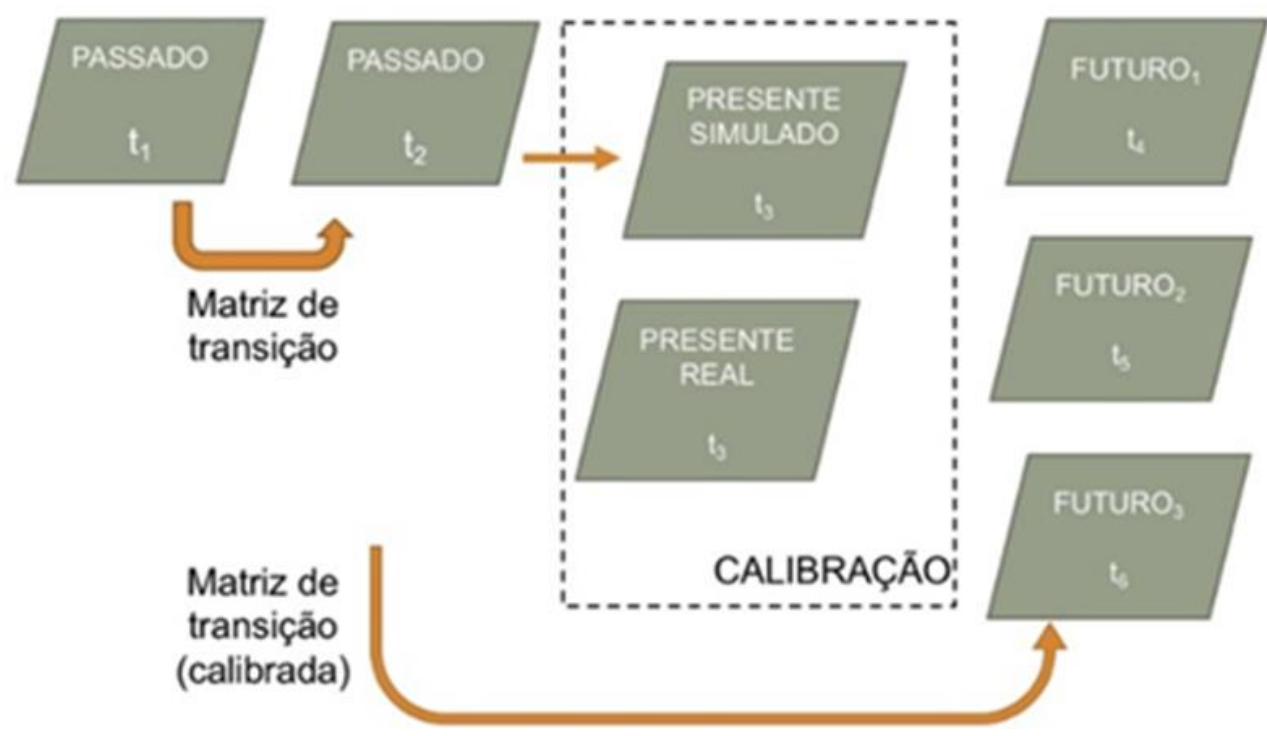

Figura 4 - Representação esquemática da calibração do modelo. Fonte: Oliveira (2019).

Com o modelo de simulação calibrado e validado, os parâmetros utilizados na calibração e a matriz de transição tornam-se confiáveis para serem replicados em cenários futuros (Figura 4). Através da simulação de cenários futuros do uso do solo foram obtidos os gráficos de tendência, que corresponderam à manutenção do mesmo padrão de crescimento de área urbanizada e diminuição de área vegetada, padrão verificado na situação real.

Assim, a partir do cenário real do ano de 2017, adotaram-se intervalos de curto e médio prazo para os cenários futuros simulados $(2020,2021,2022,2023,2024$ e 2025).

\section{Resultados}

Os mapas de uso do solo de Fortaleza - de 2009 e de 2017 - podem ser visualizados na Figura 5. Por meio dessa espacialização pode-se verificar que se trata de uma área com uso do solo predominantemente urbano. A área considerada neste estudo foi de $297,10 \mathrm{~km}^{2}$, devido à exclusão de corpos d'água, praias, dunas e áreas agropecuárias.

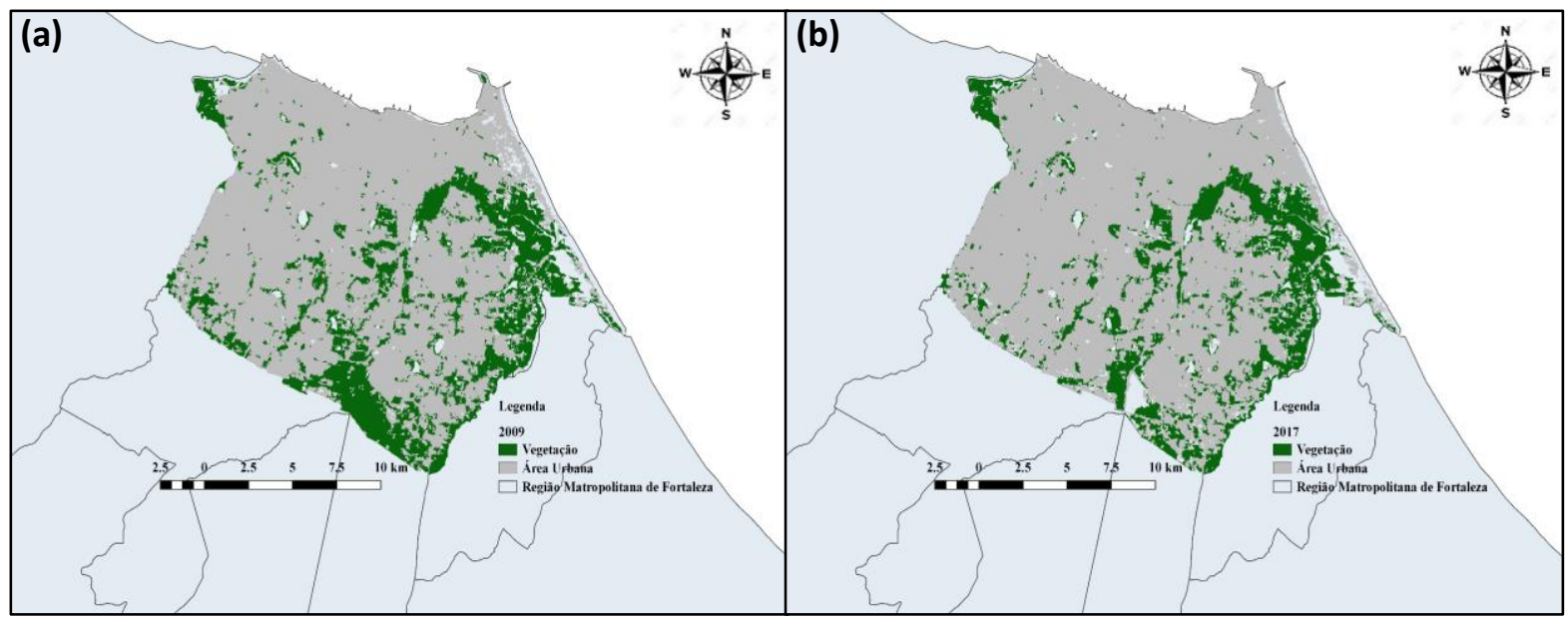

Figura 5 - (a) Representação do uso do solo de Fortaleza 2009 (b) Representação do uso do solo de Fortaleza 2017. Fonte: Autores (2020). 
O cálculo das áreas para os cenários I (2009) e II (2017) em ambiente SIG (Tabela 1) permitiu uma quantificação das tendências de mudança que haviam ocorrido no uso do solo de Fortaleza durante os oito anos. Observou-se que nesse período ocorreu um aumento de 3,93\% na área urbanizada de Fortaleza. Se essa tendência continuar ocorrendo, em 2025 (Figura 6) dos 297,10 km² de área urbanizada e vegetada, 82,43\% serão de área urbanizada e apenas 17,57\% serão de área vegetada.

Esse crescimento da área urbanizada pode ser explicado pelo aumento de pessoas vivendo em áreas urbanas. Atualmente, mais da metade da população mundial (55\%) vive em áreas urbanas. Essa proporção foi de apenas $30 \%$ em 1950, e as estimativas indicam que ela aumentará para $68 \%$ até 2050 (UN DESA, 2018).

Tabela 1 - Quantificação das áreas vegetadas e urbanizadas

\begin{tabular}{lllll}
\hline Área $\left(\mathbf{k m}^{2}\right)$ & \multicolumn{1}{l}{ Anos } & & \\
\cline { 2 - 5 } & $\mathbf{2 0 0 9}$ & & $\mathbf{2 0 1 7}$ & \\
\hline Área vegetada (1) & 71,26 & $23,83 \%$ & 59,14 & $19,90 \%$ \\
\hline Área urbanizada (2) & 227,76 & $76,17 \%$ & 237,96 & $80,10 \%$ \\
\hline
\end{tabular}

Fonte: Autores (2020).

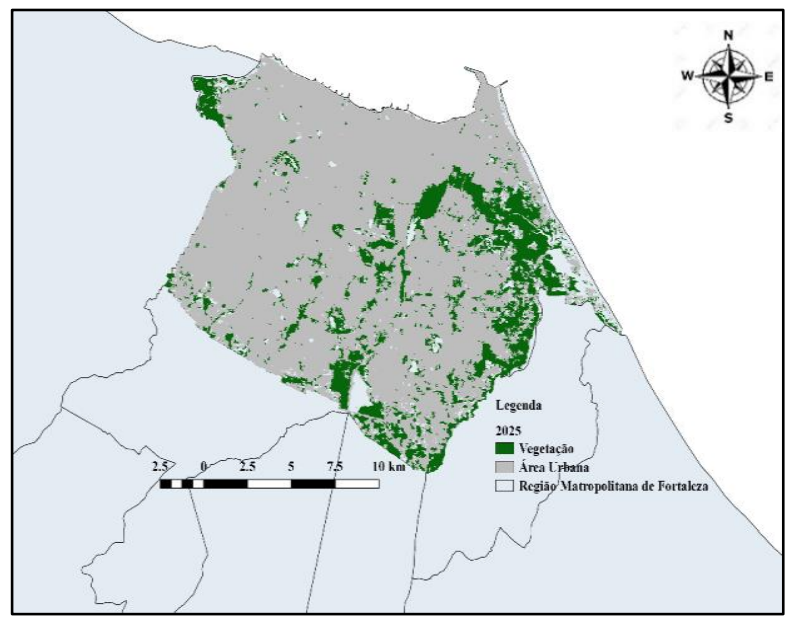

Figura 6 - Simulação do uso do solo de Fortaleza 2025. Fonte: Autores (2020).

Com base nos mapas inicial e final, foram calculadas taxas de transição em um passo simples, ou seja, do período completo (8 anos) e em passos múltiplos (anualizadas), derivada de uma matriz ergódica, isto é, uma matriz que possui auto-valores e auto-vetores reais (Soares-Filho et al., 2009). Na matriz de transição verificou-se uma tendência maior de mudança de área vegetada para área urbanizada, em vista da mudança de uso de área urbanizada para área vegetada (Tabela 2).

Tabela 2 - Matriz de transição

\begin{tabular}{lll}
\hline De (2009) & Para (2017) & Porcentagem de mudança \\
\hline 1 & 2 & $26,50 \%$ \\
\hline 2 & 1 & $3,67 \%$ \\
\hline
\end{tabular}

Fonte: Autores (2020).

A Tabela 3 apresenta os parâmetros de calibração da matriz de transição calculada inicialmente entre os mapas de entrada do modelo de simulação, garantindo que esses mesmos percentuais de mudanças pudessem ser utilizados nos mapas futuros de uso do solo simulados nesse estudo. 0 índice de isometria varia de 0 a 2 , as manchas assumem uma forma mais isométrica à medida que este número 
aumenta. 0 grau de fragmentação das manchas é inversamente proporcional ao valor do índice (Almeida et al., 2008).

Tabela 3 - Parâmetros utilizados no ajuste e execução do modelo de simulação de uso do solo

\begin{tabular}{lllll}
\hline De (2009) & Para (2017) & Tamanho médio (ha) & Variância (ha) & Isometria \\
\hline Área vegetada (1) & Área urbanizada (2) & 0,928710754 & 3,454293833 & 0,869423282 \\
\hline Área urbanizada (2) & Área vegetada (1) & 0,487021998 & 0,905032217 & 0,686351438 \\
\hline
\end{tabular}

Fonte: Autores (2020).

De acordo com os resultados dos índices V e JIU no teste de dependência espacial, nenhum par de variáveis apresentou correlação acima de $50 \%$ e, portanto, não houve exclusão de nenhuma variável.

Na validação do modelo de simulação, é aconselhável sempre escolher o menor valor de similaridade, uma vez que mapas randômicos tendem a produzir um alto ajuste inicial quando comparados univocamente, porque eles dispersam as mudanças sobre todo o mapa.

O menor valor da similaridade fuzzy entre os mapas-diferença para o modelo de simulação de uso do solo foi de 0,83 para a validação utilizando função de decaimento exponencial em janela de tamanho 11x11 (matriz de pixels de 11 linhas e 11 colunas). Isso indica um alto valor de similaridade, se comparado com Novaes et al. (2011), que sugerem que valores próximos a 0,4 indicam um bom nível de compatibilidade.

Para a validação utilizando função de decaimento constante em janelas múltiplas, foram analisados os valores das janelas de $3 \times 3$ e $5 \times 5$ pixels, onde o modelo de simulação de uso do solo obteve os valores 0,83 e 0,88 , respectivamente. Isso indica novamente um alto valor de similaridade, tendo em vista que, de modo prático, índices de similaridade fuzzy adaptados com valores oscilando entre 0,45 e 0,50 para janelas com tamanhos de 3x3 a 5x5 têm denotado concordâncias aceitáveis (Almeida et al., 2008; SoaresFilho et al., 2013).

Além da validação do próprio modelo de simulação, uma comparação direta realizada entre os mapas real e simulado do ano de 2017 através de uma subtração simples entre os mapas matriciais (formato raster) no software QGIS apresentou um grau de similaridade de 95,96\%.

Os cenários prospectivos obtidos através das simulações de mudanças de uso do solo representam as tendências de futuras configurações espaciais da cidade de Fortaleza. Aplicando uma análise de regressão linear (Figura 7) verificou-se que ao longo dos anos (y) existe uma tendência de diminuição da área vegetada (x), decorrente do aumento da área urbanizada (x) na cidade de Fortaleza. Essa tendência já tinha sido observada entre os anos 2009 e 2017 (dados de entrada do modelo de simulação).

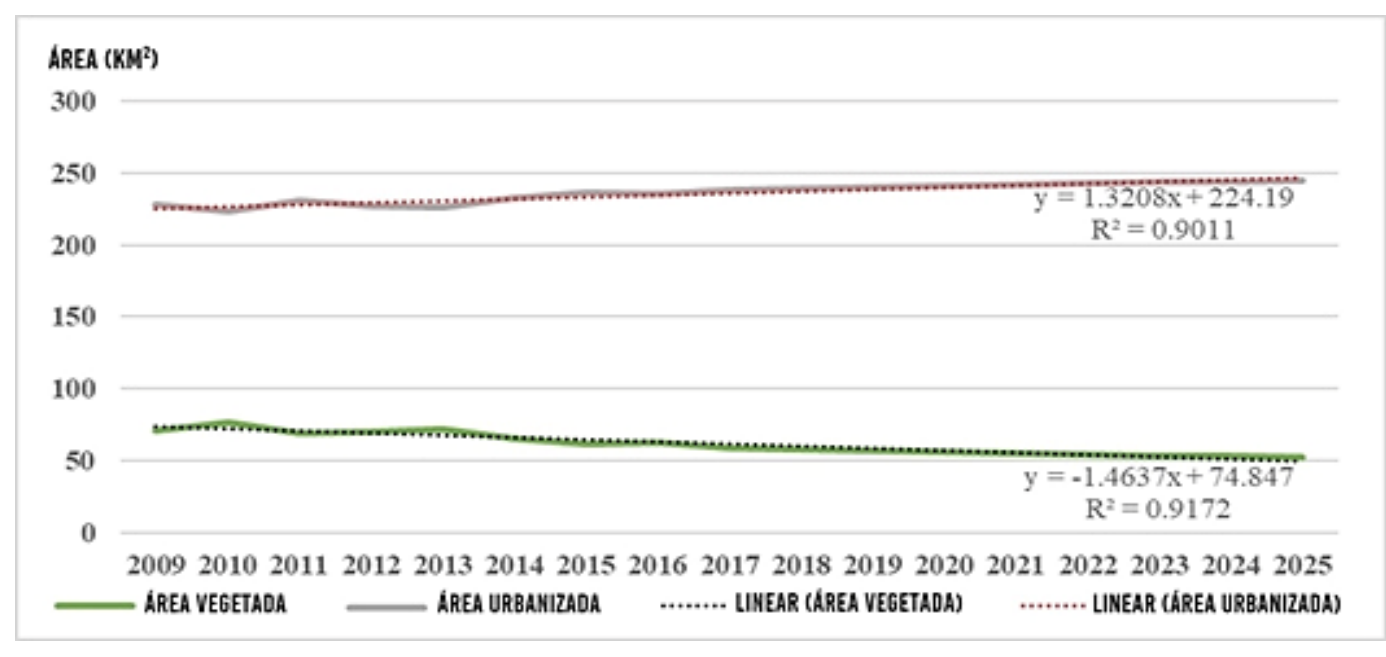

Figura 7 - Tendência do uso do solo de Fortaleza. Fonte: Autores (2020). 
Em média, 3\% de área vegetada estavam se transformando em área urbanizada por ano. Apesar de ser uma mudança anual discreta, se essa tendência de aumento continuar acontecendo, como apontou a análise de regressão linear, impactos ambientais negativos podem ser desencadeados ou agravados.

Segundo Santos (2007), a forma como é feita a ocupação do espaço urbano tem provocado sucessivos e inúmeros problemas ambientais, como degradação da cobertura vegetal, perda de biodiversidade, obstrução e alteração da rede de drenagem, transmissão de doenças de veiculação hídrica, contaminação e poluição do ar, da água e do solo, perda de terras produtivas, desencadeamento de processos erosivos, entre outros.

\section{Conclusões}

A pesquisa apresenta a possibilidade de uma modelagem preditiva do uso do solo em larga escala, que foi possibilitada pela disponibilidade de produtos de uso e ocupação do solo, como a coleção MapBiomas. Os resultados ampliam as possibilidades de modelagem urbana, hidrológica e social a partir de sistemas de células que interagem de forma complexa, assumindo processos auto-organizáveis para prever a expansão urbana. Entender a evolução da expansão urbana é imprescindível para um adequado planejamento das cidades e um melhor atendimento à população por parte dos equipamentos urbanos. Nesse sentido, o estudo verificou o crescimento da ocupação urbana por meio da combinação de autômatos celulares com as Cadeias de Markov (CA-Markov), analisando a influência da vizinhança na mudança de uma área.

Essa influência da vizinhança foi confirmada pelo fato de que o crescimento urbano aconteceu justamente em áreas que já eram muito urbanizadas. Isso fez com que áreas vegetadas que estavam cercadas por áreas urbanizadas se transformassem em espaços urbanos. Já as áreas vegetadas, em sua grande maioria áreas de proteção ambiental, se mantiveram vegetadas, como o Parque Estadual do Cocó, por exemplo.

Através da geração de mapas temáticos do uso do solo foi possível identificar que a área de estudo apresentou uma grande expansão urbana. Isso foi verificado com a comparação direta entre os mapas de entrada do modelo de simulação (2009 e 2017), em que $26,50 \%$ das áreas que antes eram vegetadas em 2009 se tornaram urbanizadas em 2017. Essa tendência de expansão urbana também se confirmou nas simulações dos cenários de prognóstico (2020, 2021, 2022, 2023, 2024 e 2025).

A partir dos dados estimados de crescimento urbano de Fortaleza para os cenários de prognóstico foi visto que em 2025 dos 297,10 $\mathrm{km}^{2}$ de área urbanizada e vegetada, 82,43\% serão de área urbanizada e apenas 17,57\% serão de área vegetada. As informações e resultados obtidos nesse estudo podem ser úteis para um planejamento estratégico de cidades, como, por exemplo, tomando medidas de delimitação de áreas passíveis de sofrer maior pressão pela ocupação indiscriminada, visando assim que os planejamentos urbano e ambiental sejam norteados pelas simulações de cenários futuros que mostrem como as mudanças irão ocorrer se o comportamento do uso do solo atual continuar acontecendo.

Nesse sentido, é importante relatar que as mudanças simuladas para cenários futuros podem não acontecer de maneira linear como mostra o estudo, caso ocorram dentro do período previsto pressões do mercado imobiliário, por exemplo, que podem nortear uma ocupação em áreas não necessariamente previstas, segundo as condições atuais. Também as mudanças nos aspectos do planejamento urbano e ambiental - como zoneamento urbano, delimitação de áreas de proteção - e mesmo políticas públicas seriam suficientes para determinar vetores de expansão e/ou áreas de estabilização.

Sabendo disso, o modelo de simulação é válido para simular cenários futuros tendo como base as condições atuais, mas ele também pode ser alimentado durante o período previsto caso situações como relatadas acima aconteçam, sendo incluídas no modelo de simulação como variáveis dinâmicas, obtendo assim um resultado futuro mais compatível com as mudanças ocorridas. 


\section{Referências}

Almeida, C. M., Gleriani, J. M., Castejon, E. F., Soares-Filho, B. S. (2008). Using neural networks and cellular automata for modelling intra - urban land - use dynamics. International Journal of Geographical Information Science, Londres, 22 (9), 943-963. https://doi.org/10.1080/13658810701731168

Almeida, C. M. (2003). Modelagem Da Dinâmica Espacial Como Uma Ferramenta Auxiliar Ao Planejamento: Simulação De Mudanças De Uso Da Terra Em Áreas Urbanas Para As Cidades De Bauru E Piracicaba (Sp), Brasil. (Tese de Doutorado) Instituto Nacional de Pesquisas Espaciais, São José dos Campos.

Almeida. M. G. \& Rosen, T. J. (1993) Desenvolvimento urbano e a questão ambiental no estado do Ceará. In Fórum Sociedade Civil Cearense sobre meio ambiente e desenvolvimento (Eds.), Diagnóstico sócio-ambiental do estado do Ceará: o olhar da sociedade civil (p. 67-115). Fortaleza: BNB.

Bonham-Carter, G. F. (1994). Geographic information systems for geoscientists: modelling with GIS. (1a. ed., Vol 13). Ontário: Pergamon.

Câmara, A. S., Ferreira, F. \& Castro, P. (1996). Spatial simulation modelling. In Fissher, M., Scholtem, H. J. \& Unwin, D. (Eds.), Spatial analytical perspectives on GIS (p. 223-235). Londres: Taylor \& Francis.

Delaneze, M. E. (2011) Modelagem dinâmica espacial aplicada na avaliação das mudanças da cobertura da terra no entorno de dutos utilizando autômatos celulares - estudo de caso duto orbel. (Dissertação de Mestrado). Universidade Estadual Paulista Júlio de Mesquita Filho, Rio Claro - SP.

Dias, L. T. \& Walde, D. H. G. (2013). Modelagem da dinâmica espacial do uso e ocupação do solo na Bacia Hidrográfica do Lago Paranoá - DF. Revista Brasileira de Cartografia, 65 (1), 77- 94.

Gonçalves, R. M., Centeno, T. M. \& Candeias, A. L. B. (2011). Cellular Automata Applied to Tendency Models in MultiTemporal Images. Revista Brasileira de Cartografia, 63 (2), 233-241.

Gong, W, Yuan L., Fan, W. \& Stott, P. (2015). Analysis and simulation of land use spatial pattern in Harbin prefecture based on trajectories and cellular automata-markov modelling. International Journal of Applied Earth Observation and Geoinformation, 34 (1), 207-216. https://doi.org/10.1016/j.jag.2014.07.005

Gorelick, N., Hancher, M., Dixon, M., Ilyushchenko, S., Thau, D. \& Moore, R. (2017) Google Earth Engine: Planetary-scale geospatial analysis for everyone. Remote Sensing of Environment, 202 (1), 18-27.

https://doi.org/10.1016/j.rse.2017.06.031

Hagen, A. (2003). Fuzzy set approach to assessing similarity of categorical maps. International Journal of Geographical Information Science, 17 (3), 235-249. https://doi.org/10.1080/13658810210157822

Instituto Brasileiro de Geografia e Estatística - IBGE (2018). Cidade e Estados. Brasil: IBGE. Recuperado em 5 de junho de 2019, de https://www.ibge.gov.br/informacoes-por-cidade-e-estado.html?t=destaques\&c=2504009.

Instituto de Planejamento de Fortaleza - IPLANFOR (2015). Série Fortaleza 2040. Recuperado em 10 de junho de 2019 , de http://fortaleza2040.fortaleza.ce.gov.br/site/

Jat, M. K., Choudhary, M. \& Saxena, A. (2017). Urban Growth Assessment and Prediction Using RS, GIS and SLEUTH Model for a Heterogeneous Urban Fringe. The Egyptian Journal of Remote Sensing and Space Science, 20 (2), $223-241$. https://doi.org/10.1016/j.ejrs.2017.02.002

Johansson, A., Batty, M., Hayashi, K., Al Bar, O., Marcozzi, D. \& Memish. Z. A. (2012). Crowd and environmental management during mass gatherings. The Lancet Infectious Diseases, 12 (2), 150-156. https://doi.org/10.1016/S1473-3099(11)70287-0

Li, X., Zhou, Y., Asrar, G. R., Imhoff, M. \& Li, X. (2017). The surface urban heat island response to urban expansion: a panel analysis for the conterminous United States. Science of The Total Environment, 605-606 (15), 426-435.

https://doi.org/10.1016/j.scitotenv.2017.06.229

Liu, F., Zhang, Z., Zhao, X., Wang, X., Zuo, L., Wen, Q., Yi, L., Xu, J., Hu, S. \& Liu, B. (2019). Chinese cropland losses due to urban expansion in the past four decades. Science of The Total Environment, 650 (10), 847-857.

https://doi.org/10.1016/j.scitotenv.2018.09.091.

Maeda, E. E., Almeida, C. M., Ximenes, A. C., Formaggio, A. R., Shimabukuro, Y. E. \& Pellika, P. (2011). Dynamic modeling of forest conversion: Simulation of past and future scenarios of rural activities expansion in the fringes of the Xingu 
National Park, Brazilian Amazon. International Journal of Applied Earth Observation and Geoinformation, 13 (3), $435-446$. https://doi.org/10.1016/j.jag.2010.09.008.

Malleson, N. \& Birkin, M. (2011). Towards victim-oriented crime modelling in a social science e-infrastructure. Philosophical Transactions of the Royal Society: Mathematical, Physical and Engineering Sciences, 369 (1949), 33533371. https://doi.org/10.1098/rsta.2011.0142.

Martins, C. M. T., Mendes, M. G. T., Abreu J. M., Almeida, J. P. L. de, Lima J. P. de \& Lima, I. P. de. (2010). Hidrologia urbana: Conceitos básicos (1a. ed.). Lisboa: Entidade Reguladora dos Serviços de Águas e Resíduos.

Mas, J. F., Kolb, M., Houet, T., Paegelow, M. \& Olmedo, M. T. C. (2014). Inductive pattern-based land use/cover change models: A comparison of four software packages. Environmental Modelling and Software, 51 (1), 94-111. https://doi.org/10.1016/j.envsoft.2013.09.010.

Matthews, R. B., Girbert, N., Roach, A., Polhill, J. G., \& Gotts, N. (2007). Agent-based land-use models: a review of applications. Landscape Ecology, 22 (10), 1447-1459. https://doi.org/10.1007/s10980-007-9135-1.

Meirelles, M. S. P., Camara, G. \& Almeida, C. M. (2007) Geomática: modelos e aplicações ambientais (1a. ed.). Brasília - DF: Embrapa Informação Tecnológica.

Novaes, M. R., Almeida C. M.; Rudorff, B. F. T. \& Aguiar, D. A. (2011) Cenários prognósticos baseados em modelagem dinâmica espacial para o manejo da colheita da cana-de-açúcar no estado de São Paulo. In Anais do XV Simpósio Brasileiro de Sensoriamento Remoto (p. 407-414). Curitiba: SBSR.

Oliveira, L. M. (2019) Modelagem dinâmica e cenários urbanos de demanda de água: simulações em Campina Grande - P. (Dissertação de mestrado). Programa de Pós-Graduação em Engenharia Civil e Ambiental, Universidade Federal de Campina Grande, Campina Grande.

Rossetti, L. A. F. G., Almeida, C. M. \& Pinto, S. A. F. (2013). Análise de mudanças no uso do solo urbano e rural com a aplicação de modelagem dinâmica espacial. In Anais do XVI Simpósio Brasileiro de Sensoriamento Remoto (p. 6316-6323). Foz do Iguaçu: SBSR.

Roy, G. G. \& Snickars, F. (1996). Citylife: a study of cellular automata in urban dynamics. In Fisher, M, Scholten, H. J. \& Unwin, D. (Eds.). Spatial Analytical Perspectives on GIS (p. 236-254). Londres: Taylor \& Francis.

Rubira, F. G. (2016). Análise multitemporal da expansão urbana de Maringá-PR durante o período de 1947 a 2014 envolvendo o Parque Municipal do Cinquentenário e as principais áreas verdes do município. Caderno de Geografia, 26 (46), 333-361. https://doi.org/10.5752/P.2318-2962.2016v26n46p333.

Sakieh, Y., Amiri, B. J., Danekar, A., Feghhi, J. \& Dezhzam, S. (2015). Simulating urban expansion and scenario prediction using a cellular automata urban growth model, SLEUTH, through a case study of Karaj City, Iran. Journal of Housing and the Built Environment, 30 (4), 591-611. https://doi.org/10.1007/s10901-014-9432-3.

Santos, C. Z. (2007) Alterações socioambientais na bacia hidrográfica do ribeirão do Lajeado no município de Paraibuna SP (Dissertação de Mestrado). Instituto de Pesquisa e Desenvolvimento, Universidade do Vale do Paraíba, São José dos Campos.

Soares-Filho, B. S., Cerqueira, G. C. \& Pernnanchin, C. L. (2002). DINAMICA - A stochastic cellular automata model designed to simulate the landscape dynamics in an Amazonian colonization frontier. Ecological Modelling, 154 (3), $217-$ 235. https://doi.org/10.1016/S0304-3800(02)00059-5.

Soares-Filho, B. S.,Rodrigues, H. O. \& Costa, W. L. (2009) Modelagem de Dinâmica Ambiental com Dinamica EGO (1a. ed.). Belo Horizonte - MG: Centro de Sensoriamento Remoto - Universidade Federal de Minas Gerais.

Soares-Filho, B. S., Rodrigues, H. \& Follador, M. (2013). A hybrid analytical-heuristic method for calibrating land-use change models. Environmental Modelling and Software, 43 (1), 80-87. https://doi.org/10.1016/j.envsoft.2013.01.010

Souza, C. \& Azevedo, T. (2017) MapBiomas General "Handbook": Algorithm: Theoretical Basis Document (ATBD) (1a. ed.). São Paulo.

Stanganini, F. N. \& Lollo, J. A. (2018). The growth of the urban area of São Carlos/SP between the 2010 and 2015: the advancement of environmental degradation. Brazilian Journal of Urban Management, 10 (1), 118-128.

https://doi.org/10.1590/2175-3369.010.supl1.ao14. 
United Nations Department of Economic and Social Affairs - UN DESA (2018). World Urbanization Prospects. Recuperado em 10 de junho de 2019, de https://population.un.org/wup/Publications/Files/WUP2018-Report.pdf.

Vick, E. P. \& Bacani, V. M. (2019). Prediction of the future scenario of the expansion of areas of forestry into the brazilian cerrado using a cellular automata Markov Chain model. Mercator - Revista Eletrônica de Geografia da Universidade Federal do Ceará, 18. https://doi.org/10.4215/rm2019.e18026.

Votsis, A. (2017). Utilizing a cellular automaton model to explore the influence of coastal flood adaptation strategies on Helsinki's urbanization patterns. Computers, Environment and Urban Systems, 64 (1), 344-355.

https://doi.org/10.1016/j.compenvurbsys.2017.04.005.

Editor responsável: Fábio Duarte

Submissão: 26 mar. 2020

Aprovação: 10 out. 2020 